\section{Case Reports in Oncology}

\title{
Unilateral Ptosis in an Older Adult; a Rare Presentation of Primary Extranodal Hodgkin's Lymphoma
}

\author{
Insija llyas Selene ${ }^{a} \quad$ Jemin Aby Jose ${ }^{b} \quad$ Abdur Rehman Jamila \\ Roma Srivastava $^{c}$ Weichen Wang ${ }^{d}$
}

aDivision of Internal Medicine, Central Michigan University, Saginaw, MI, USA; bivision of Internal Medicine, Carle Foundation Hospital, Urbana, IL, USA; 'Division of Hematology \&

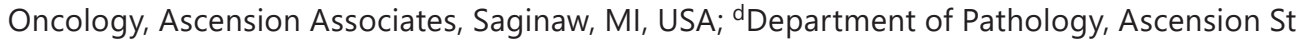
Mary's Hospital, Saginaw, MI, USA

\author{
Keywords \\ Hodgkin lymphoma $\cdot$ Hodgkin's disease $\cdot$ Extranodal Hodgkin lymphoma $\cdot$ Orbit
}

\begin{abstract}
Hodgkin lymphoma $(\mathrm{HL})$ is a neoplasm arising from $B$ cells characterized by the presence of Reed-Steenberg cells. Primary extranodal presentation is rare and accounts for less than $1 \%$ of all $\mathrm{HL}$ cases. In addition, the orbit is an uncommon site of extranodal $\mathrm{HL}$, with only 9 cases reported in the literature. We present a case of an 84-year-old male who presented with right eye ptosis. He was diagnosed with stage IIE Orbital HL and treated with combined modalities of radiation and chemotherapy. He continues to be in complete remission after 1 year of therapy. Hodgkin's disease has an excellent prognosis, and recent data show it is curable in at least $80 \%$ of the patients. Extranodal involvement represents systemic dissemination of Hodgkin's disease in most cases and is usually considered an advanced-stage disease with a poor prognosis. In rare circumstances, extranodal involvement can be the primary manifestation. Unfortunately, there are only a few case reports and case series regarding this topic. We attempt to add another case to the literature emphasizing the prognosis and outcome of primary extranodal $\mathrm{HL}$.
\end{abstract}




\section{Introduction}

Hodgkin's lymphoma (HL) is a lymphoproliferative disorder usually involving the reticuloendothelial system: commonly the cervical, mediastinal, and axillary lymph nodes [1-3]. It represents approximately $9.9 \%$ of newly diagnosed Lymphoma in the USA [4]. HL can affect people of all ages but mostly involves people between 20 and 40 years and those over 55 years. HL is classified into classical HL (CHL -95\%) and nodular lymphocyte-predominant HL $(-5 \%)[1,2]$. An extranodal presentation of HL is unusual and accounts for less than $1 \%$ of all cases $[2,3,5]$. The most common sites for extranodal presentation are the stomach, liver, lungs, intestine, and bones $[2,3,6-8]$. This case describes a rare primary extranodal presentation of HL.

\section{Case Report}

An 84-year-old male with a history of chronic obstructive pulmonary disease presented with 2.5 months of progressive right eye droop. He denied any changes in visual acuity, photophobia, painful eye movements, or systemic symptoms such as fever, night sweats, or weight loss. Medical and social history was noncontributory, with no prior history of radiation and malignancy with no suggestion of an immunodeficient state. Physical exam revealed right eye ptosis with no extraocular motility limitation and no abnormality in the slit lamp exam. There was no evidence of organomegaly or lymph node involvement. Magnetic resonance imaging (MRI) of the orbit and brain showed a mass in the right orbit with involvement of the right frontal sinus and extending to the anterior cranial fossa (Fig. 1a, b). A whole-body PET-CT scan demonstrated hypermetabolic activity in the medial aspect of the right orbit and right frontal sinus (Fig. 2). The excisional biopsy with immunohistochemical staining of orbital mass was positive for nodular sclerosing HL (Fig. 3a, b). The disease was classified as earlystage (Stage IIE, unfavorable) based on Ann Arbor staging due to the localized involvement of extra-lymphatic orbital tissue. A 2D echocardiogram and pulmonary function test to assess baseline cardiac status and respiratory reserve were within normal limits. The International Prognostic Score (IPS) of our patient was 2.

He received Adriamycin, Bleomycin, Vinblastine, and Dacarbazine (ABVD) with the involved site radiation therapy (ISRT) per NCCN guidelines. The patient initially refused ISRT due to
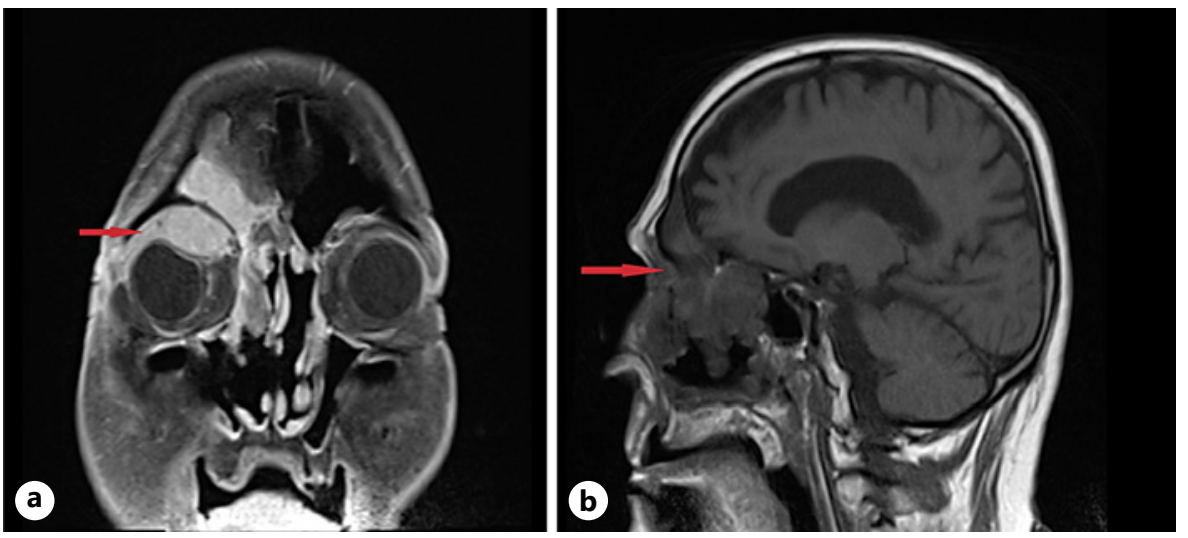

Fig. 1. a T1 weighted coronal MRI showing hypointense orbital lesion. b T2 weighted sagittal MRI image of Orbital soft tissue. 


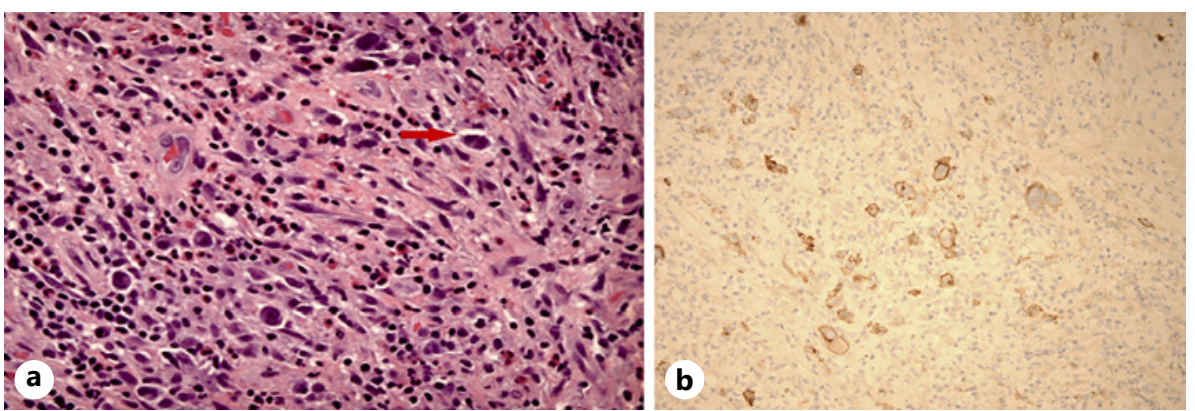

Fig. 2. Fused Trans CT images of PET-CT skull base/mid-thigh level showing focal soft tissue mass medial aspect of right orbit with maximum SUV reading of 12 .

Fig. 3. a CHL (H\&E stain, original magnification $\times 500)$. b CHL (Immunohistochemical stain demonstrating CD30 positive Reed-Sternberg cells, original magnification $\times 500$ ).

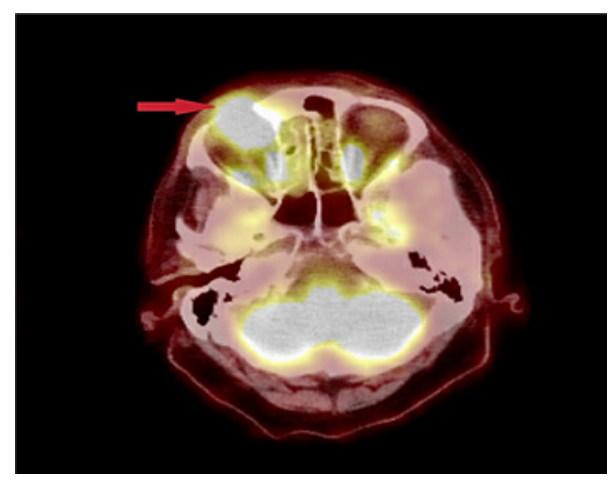

Fig. 4. Follow-up PET-CT image - resolution of right orbitalFDG avid mass.

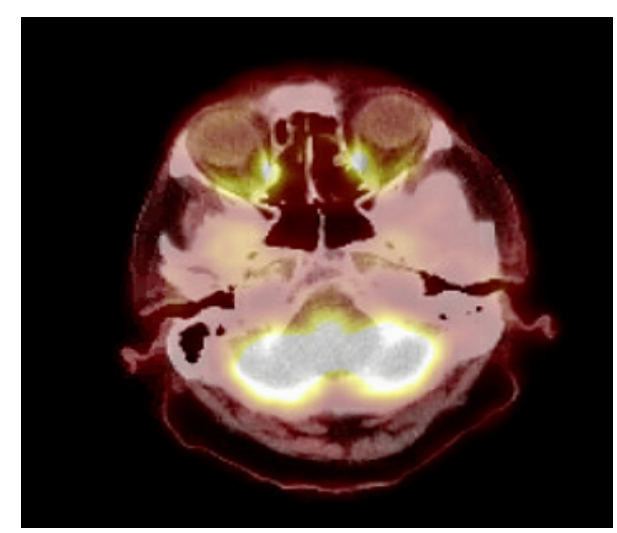

concerns of radiation-induced side effects. A PET-CT scan to assess the response after two cycles of chemotherapy showed a complete response (CR). While on treatment, he experienced worsening dyspnea with a repeat pulmonary function test showing significantreduction in the diffusion capacity and severe obstructive defect. Because of compromised PFT, we discontinued bleomycin, and he received an additional two cycles of the AVD regimen followed by 20 Gy (Gy) of ISRT. After four treatment cycles, PET-CT (Fig. 4) showed CR with complete orbital-FDG avid mass resolution. He remains under surveillance as per NCCN guidelines and continues to be in CR. 


\section{Discussion}

HL presents with painless lymphadenopathy with or without B symptoms of fever, chills, night sweats, and un explained weight loss of $>10 \%$ body weight [1]. Primary extranodal Hodgkin's disease is an uncommon presentation of HL, with most of the published studies being case reports. Most of the primary extranodal HL affects the younger age-group $(<55$ yrs $)[1,2]$. There are no identified risk factors for the development of HL, but familial factors, viral exposure, and immune suppression can play a role in pathogenesis [1].

When HL arises from an extra-lymphatic organ, clinical presentation varies based on site of origin. Even though Orbital lymphomas are the most common primary orbital tumor (50-60\%) in elderly patients, the primary extranodal presentation of HL in Orbit is rare [3-5]. Ma et al. [6] provide insight into clinical characteristics of patients diagnosed with primary extranodal HL. He identified 26 patients with primary extranodal HL among 251 HL patients (1998-2012), and the site of involvement was lung (42.3\%), stomach and intestine (23.1\%), liver (19.2\%), bones (15.4\%), and thyroid (3.8\%) [6]. Another literature review by Li et al. [7] (1995-2012) identified around 9 cases of HL with primary osseous involvement. A retrospective analysis of 281 PET/CT studies to look for extranodal involvement in Lymphoma by Das et al. [8] in 2014 described similar distribution. Another study by Iyengar et al. [9] on HL involving head and neck region identified 8 cases originating from thyroid. None of the above case series mentioned orbital HL, and an extensive literature search identified only 9 cases reported as described in Table 1.

The diagnosis of Orbital HL needs excision biopsy and immunohistochemical staining $[1,6,7,9]$. The two histological subtypes reported in case reports of Orbital HL are nodular sclerosis (46.2\%) and mixed cellularity (38.5\%) [10-13]. Inflammatory markers such as ESR and LDH could be elevated and serve as a valuable marker of disease response [1]. In addition, CT or MRI of the orbit is necessary to identify the size and anatomical location of the tumor $[3,4,7,8,17]$. Even though CT, MRI help identify anatomical locations of lymphoma, it can get tricky in scenarios where there is an invasion of adjacent structures $[3,5,8]$. The PET-CT scan interpretation used for the initial diagnosis and staging of $\mathrm{HL}$ is also challenging when the lesion is near the brain, especially in orbit where radiotracer uptake is high $[18,19]$.

Ann Arbor staging systems have been in use since 1989 for HL's clinical staging, with recent modifications in descriptive terminology with the addition of extranodal sites [20,21]. However, this staging system is less reliable in ocular adnexal Lymphoma, where the orbit is the only extranodal location, and they get classified as stage I-IIE regardless of the extent of adjacent tissue involvement [3]. The TNM staging system overcame the limitation with sitespecific information and precise definition of the tumor extent and the local spread, but currently used only in Non-HL [3-5].

The treatment options for HL include chemotherapy and radiation with newer additions, including immunotherapy and targeted therapy based on staging according to NCCN guidelines [22,23]. Elderly patients have a higher degree of adverse events with chemotherapeutic regimens; hence, current studies consider dose attenuation in early-stage disease [23]. Although HL is a chemo responsive tumor, we can consider debulking surgery in orbital involvement with significant eye symptoms $[3,4,11,13]$.

Patients with an early-stage HL have excellent cure rates of $>90 \%$, so the focus of the studies in the last 10 years is to find a regimen with maximum efficacy while minimizing toxicity [24, 25]. For example, the German HD10 trial investigated different cycles of chemotherapy and different intensities of ISRT in early-stage favorable CHL and found two cycles of ABVD followed by 20 Gy of ISRT as the optimal approach [25]. Multiple studies, including clinical trials and meta-analysis, showed better overall survival and progression-free survival with the treatment adaptation strategies using interim PET-CT [26-28]. The need for consolidation

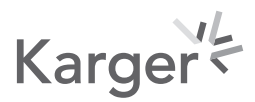


Case Reports in Oncology
Case Rep Oncol 2022;15:106-113 DOI: 10.1159/000520715

(c) 2022 The Author(s). Published by S. Karger AG, Basel www.karger.com/cro

Selene et al.: Primary Extranodal Hodgkin's Lymphoma of Orbit

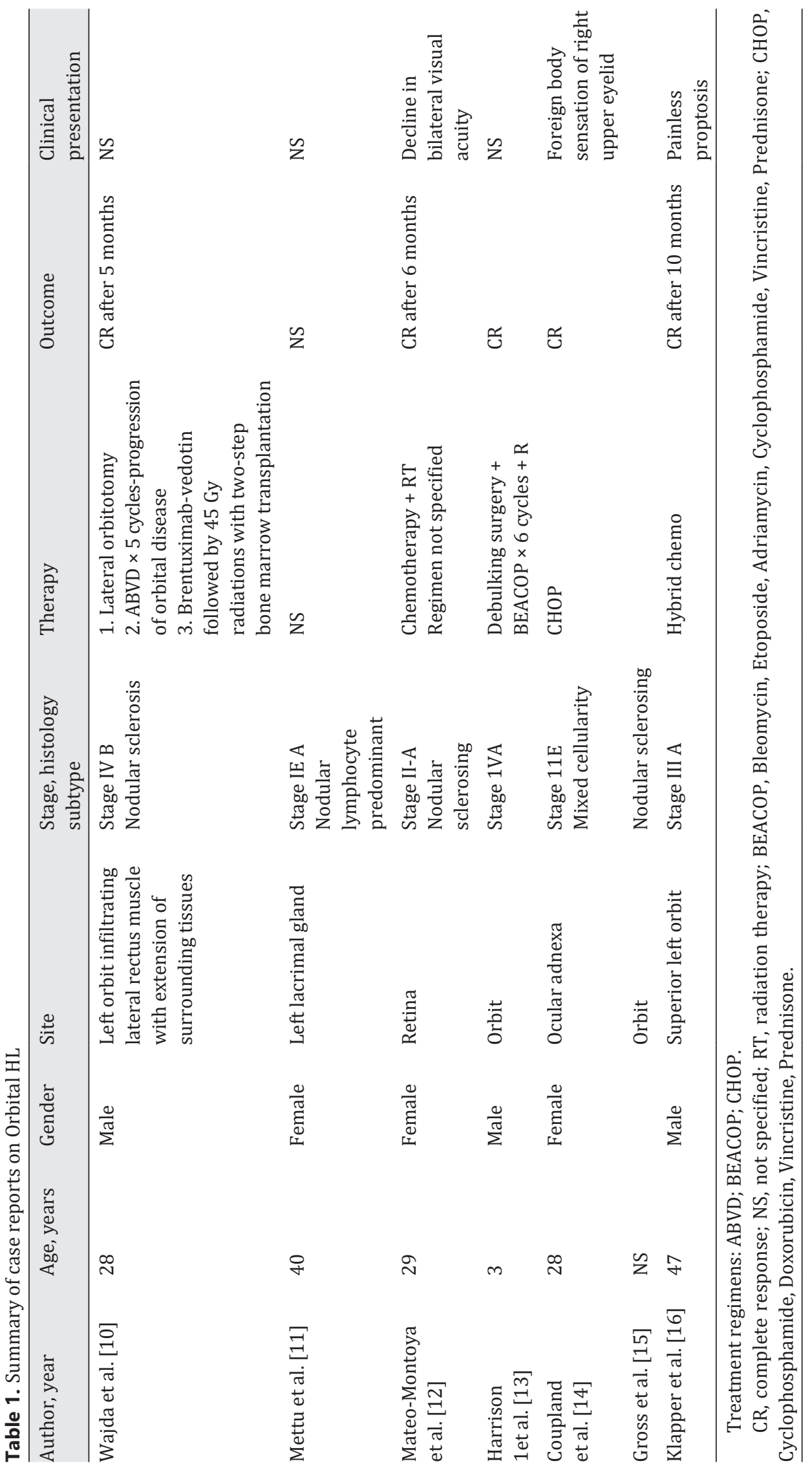

Karger' 
radiation therapy with three cycles of ABVD regimen using an integrated PET-CT scan is discussed in detail in the RAPID trial. The study concluded noninferiority of chemotherapy alone after negative PET findings compared to a consolidation radiation therapy after the chemo regimen [27]. Another study, EORTC (European Organization for Research and Treatment of Cancer) H10 trial, looked into the omission of ISRT in patients attaining negative PET scan after two ABVD cycles, which got terminated due to the rapid progression of the disease [26]. The interim PET-guided treatment response with five-point scale (5-PS) Deauville criteria is currently incorporated in NCCN guidelines to assess treatment response [21, 22, 29]. There is a need for further studies to analyze the prognosis and outcomes when HL presents with isolated extranodal involvement.

IPS-7, one of the prognostic scoring tools widely applied for HL, is limited to advancedstage disease, and no tools were taken into consideration when the disease presents only in extranodal sites $[1,6,29]$. Ma et al. [6] devised a scoring tool involving the following variables in their study on extranodal HL patients, including LDH, albumin, clinical stage, and pathological types, to define the prognosis and categorized patients into low risk and high risk. The above study showed low risk patients (score $<2$ ) had a higher 5-year overall survival (OS) as well as diseasefree survival (DFS) $(100 \%$ vs. $67.4 \%, p=0.016: 100 \%$ vs. $67.1 \%, p=0.011)$. Our patient falls into low risk based on this scoring system and has a good OS and DFS. Similarly, the other studies or case reports made individualized approaches for scoring and classification because of no definite consensus on this topic.

\section{Conclusion}

In summary, lymphoproliferative disorder affecting the orbit is usually a non-Hodgkin subtype. Primary extranodal HL is a known rare entity with orbital involvement being infrequent. The staging and prognosis of primary extranodal Hodgkin disease though less favorable than CHL, our literature review reveals that patients respond well to treatment with long-term survival.

\section{Statement of Ethics}

Ethics approval was not required for the study. A written informed consent was obtained from the patient for publication of the details of his medical case and accompanying images.

\section{Conflict of Interest Statement}

The authors have no conflict of interest to declare.

\section{Funding Sources}

There is no funding received for this manuscript.

\section{Author Contributions}

Supervised by Dr. Roma Srivastava. Patient was seen by Dr. Roma Srivastava. Report was written by Dr. Insija Ilyas Selene, Dr. Jemin Aby Jose, and Dr. Abdur Rehman Jamil.

\section{Karger's}




\section{Data Availability Statement}

All data generated or analysed during this study are included in this article. Further enquiries can be directed to the corresponding author.

\section{References}

1 Shanbhag S, Ambinder RF. Hodgkin lymphoma: a review and update on recent progress. CA Cancer J Clin. 2018 Mar;68(2):116-32.

2 Siegel RL, Miller KD, Jemal A. Cancer statistics, 2016. CA Cancer J Clin. 2019 Jan;66(1):7-30.

3 Olsen TG, Heegaard S. Orbital lymphoma. Surv Ophthalmol. 2019 Jan 1;64(1):45-66.

4 Wanyura H, Uliasz M, Kaminski A, Samolczyk-Wanyura D, Smolarz-Wojnowska A. Diagnostic difficulties and treatment of non-Hodgkin lymphoma of the orbit. J Craniomaxillofac Surg. 2007 Jan 1;35(1):39-47.

5 White VA. Understanding and classification of ocular lymphomas. Ocul Oncol Pathol. 2019;5(6):37986.

6 Ma J, Wang Y, Zhao H, Liu S, Li Q, Lin L, et al. Clinical characteristics of 26 patients with primary extranodal Hodgkin lymphoma. Int J Clin Exp Pathol. 2014;7(8):5045.

7 Li Y, Qin Y, Zheng L, Liu H. Extranodal presentation of Hodgkin's lymphoma of the sternum: a case report and review of the literature. Oncol Lett. 2018 Feb 1;15(2):2079-84.

8 Das J, Ray S, Sen S, Chandy M. Extranodal involvement in lymphoma - a pictorial essay and retrospective analysis of 281 PET/CT studies. Asia Ocean J Nucl Med Biol. 2014;2(1):42.

9 Iyengar P, Mazloom A, Shihadeh F, Berjawi G, Dabaja B. Hodgkin lymphoma involving extranodal and nodal head and neck sites: characteristics and outcomes. Cancer. 2010 Aug 15;116(16):3825-9.

10 Wajda BN, Rabinowitz MP. A rare case of orbital hodgkin lymphoma demonstrating therapeutic response to a novel systemic medication. Orbit. 2017 Jan 2;36(1):52-4.

11 Mettu P, Griffith M, Yohe S, Harrison AR. Nodular lymphocyte predominant Hodgkin lymphoma presenting with unilateral orbital involvement. Ophthalmic Plast Reconstr Surg. 2017 Mar 1;33(2):e29-31.

12 Mateo-Montoya A, Bonnel S, Wolff B, Héron E, Sahel JA. White dots in the eye fundus revealing Hodgkin's lymphoma. Eye. 2010 May;24(5):934-7.

13 Harrison AR, Mokhtarzadeh A, Larson JS, Dewan MA, Lee MS. Primary orbital manifestation of Hodgkin lymphoma in a 3-year-old child. Ophthalmic Plast Reconstr Surg. 2010 Nov 1;26(6):494-6.

14 Coupland SE, Hummel M, Stein H. Ocular adnexal lymphomas: five case presentations and a review of the literature. Surv Ophthalmol. 2002 Sep 1;47(5):470-90.

15 Gross ND, Wilson DJ, Dailey RA, Beckstead JH, Braziel RM. Nodular sclerosing Hodgkin disease with primary manifestation in the orbit. Ophthalmic Plast Reconstr Surg. 1998 May 1;14(3):169-73.

16 Klapper SR, Jordan DR, McLeish W, Pelletier C. Unilateral proptosis in an immunocompetent man as the initial clinical manifestation of systemic Hodgkin disease. Ophthalmology. 1999 Feb 1;106(2):338-41.

17 Nikolica G, Badnjar Z, Cadjenovic T, Raceta-Masic D. Primary extra nodal Hodgkin disease: bone presentation. Pol J Radiol. 2014;79:91.

18 Borkar AU, Jain K, Jain VK. A large primary orbital lymphoma with proptosis: a case report and review. Surg Neurol Int. 2018;9:249.

19 Berriolo-Riedinger A, Becker S, Casasnovas O, Vander Borght T, Édeline V. Role of FDG PET-CT in the treatment management of Hodgkin lymphoma. Cancer Radiother. 2018 Sep 1;22(5):393-400.

20 Lister TA, Crowther D, Sutcliffe SB, Glatstein E, Canellos GP, Young RC, et al. Report of a committee convened to discuss the evaluation and staging of patients with Hodgkin's disease: cotswolds meeting. J Clin Oncol. 1989 Nov; 7(11):1630-6.

21 Cheson BD, Fisher RI, Barrington SF, Cavalli F, Schwartz LH, Zucca E, et al. Recommendations for initial evaluation, staging, and response assessment of Hodgkin and non-Hodgkin lymphoma: the Lugano classification. J Clin Oncol. 2014;32:3059-68.

22 Hoppe RT, Advani RH, Ai WZ, Ambinder RF, Armand P, Bello CM, et al. Hodgkin lymphoma, version 2.2020, NCCN clinical practice guidelines in oncology. J Natl Compr Canc Netw. 2020 Jun 1;18(6):755-81.

23 Carter J, David KA, Kritharis A, Evens AM. Current treatment options for older patients with hodgkin lymphoma. Current Treat Options Oncol. 2020 May;21(5):1-8.

24 Engert A, Plütschow A, Eich HT, Lohri A, Dörken B, Borchmann P, et al. Reduced treatment intensity in patients with early-stage Hodgkin's lymphoma. N Engl J Med. 2010 Aug 12;363(7):640-52.

25 Eich HT, Diehl V, Görgen H, Pabst T, Markova J, Debus J, et al. Intensified chemotherapy and dose-reduced involved-field radiotherapy in patients with early unfavorable Hodgkin's lymphoma: final analysis of the German Hodgkin Study Group HD11 trial. J Clin Oncol. 2010 Sep 20;28(27):4199-206.

26 Raemaekers JM, André MP, Federico M, Girinsky T, Oumedaly R, Brusamolino E, et al. Omitting radiotherapy in early positron emission tomography-negative stage I/II Hodgkin lymphoma is associated with an increased risk of early relapse: clinical results of the preplanned interim analysis of the randomized EORTC/LYSA/FIL H10 trial. J Clin Oncol. 2014 Apr 20;32(12):1188-94. 
27 Barrington SF, Mikhaeel NG, Kostakoglu L, Meignan M, Hutchings M, Müeller SP, et al. Role of imaging in the staging and response assessment of lymphoma: consensus of the International Conference on Malignant Lymphomas Imaging Working Group. J Clin Oncol. 2014 Sep 20;32(27):3048.

28 Aldin A, Umlauff L, Estcourt LJ, Collins G, Moons KG, Engert A, et al. Interim PET-results for prognosis in adults with Hodgkin lymphoma: a systematic review and meta-analysis of prognostic factor studies. Cochrane Database Syst Rev. 2019;9(9):CD012643.

29 Diefenbach CS, Li H, Hong F, Gordon LI, Fisher RI, Bartlett NL, et al. Evaluation of the International Prognostic Score (IPS-7) and a Simpler Prognostic Score (IPS-3) for advanced Hodgkin lymphoma in the modern era. Br J Haematol. 2015 Nov;171(4):530-8. 